\title{
La sublimación erótico-estética de la mujer en los imaginarios simbólicos de Gabriele D’Annunzio y Amado Nervo: dos cuentos fantásticos
}

Francesca Federico ${ }^{1}$

Resumen. El presente trabajo de investigación propone una lectura comparada de dos cuentos fantásticos: L'uomo che rubò la "Gioconda" de Gabriele D’Annunzio y "La Diablesa" de Amado Nervo. El estudio tiene el propósito de destacar la confraternización de imaginarios simbólicos que hermana a los dos autores a la hora de representar a la Mujer Fatal.

Palabras clave: Mujer Fatal; Quimera; imaginarios simbólicos; Gabriele D’Annunzio; Amado Nervo; cuentos fantásticos.

[en] The Erotic and Aesthetic Sublimation of Woman in the Symbolic Imageries of Gabriele D’Annunzio and Amado Nervo: Two Fantastic Tales

\begin{abstract}
The project proposes a comparative analysis of two fantastic tales: L'uomo che rubò la "Gioconda" by Gabriele D'Annunzio and "La Diablesa" by Amado Nervo. The study investigates the fraternization of symbolic imageries that connects these authors in the characterization of the Femme Fatale.
\end{abstract}

Keywords: Femme Fatale; Chimera; symbolic imageries; Gabriele D’Annunzio; Amado Nervo; fantastic tales.

Sumario: 1. Palabras preliminares. 2. Gabriele D’annunzio fantástico: El hombre que robó la “Gioconda”. 3. Amado Nervo fantástico: "La Diablesa". 4. Cierre.

Cómo citar: Federico, F. (2020) La sublimación erótico-estética de la mujer en los imaginarios simbólicos de Gabrielle D’Annunzio y Amado Nervo: dos cuentos fantásticos, en Anales de Literatua Hispanoamericana 49, 163-167.

\section{Palabras preliminares}

Sería arriesgado proponer una lectura comparada de dos cuentos fantásticos de autores como Gabriele D’Annunzio y Amado Nervo sin proporcionar a los lectores unas premisas esenciales.

Franco Meregalli (647-648) considera relevante subrayar que la influencia ejercida por el "Vate" italiano sobre los modernistas hispanoamericanos debía atribuirse a dos razones fundamentales: su éxito en París y el favor de Rubén Darío, cuya obra de promoción facilitó al abrucés una formidable notoriedad en todo el continente americano.

En el caso específico de Amado Nervo y las posibilidades de "contagio dannunziano" debemos recordar, en primer lugar, su estancia en París desde 1900 hasta 1905. Nervo llega a Francia justo en los años en los que D'Annunzio goza de una considerable fortuna: es aclamado por el público, por la prensa y por la crítica. Y, aunque el "voluntario exilio" del italiano en París es sucesivo a esas fechas y es muy poco probable que coincidieran personalmente, consta que Nervo frecuentó el mismo ambiente literario, relacionándose con Paul Verlaine, Jean Moréas, Oscar Wilde, Leopoldo Lugones, Guillermo Valencia y, nuevamente, con el mismo Rubén Darío, con quien había trabajado en México. No se pase por alto que Valencia y Lugones, además de conocer y apreciar a D'Annunzio, tradujeron algunos de sus textos. 
Añádase a todo esto un detalle que no deja ni una sombra de incertidumbre sobre el conocimiento de la obra del "Vate" por parte de Nervo: una breve crónica de este en la cual podemos apreciar algunas valoraciones positivas y otras algo menos generosas sobre el italiano. Demos paso a su lectura:

Nadie duda de que il signor Gabrielle d'Annunzio tiene talento. Basta recordar el espiritual regalo de sus Vírgenes de las Rocas, que pasan, ya delicadas, ya austeras y melancólicas, como a través de un otoñal ensueño. Basta recordar la sabia fiebre del Inocente y de su Triunfo de la Muerte y la poderosa elegancia y la pasión arcaica de su Francesca. [...] Pero il signor Gabrielle d'Annunzio ha resuelto algo más que tener talento y auno algo más que tener mucho talento: ha resuelto tener genio, así, a secas, y ha decidido algo peor todavía: decirlo urbi et orbe.

Amado Nervo parece estar fascinado por D'Annunzio. En parte es cierto, no niega su valía como escritor. No obstante, no puede evitar criticar su pose soberbia y apunta: "Todo su talento de adjetivador, todos los ardores de su cálido estilo, todas las tersuras de algunas de sus páginas lapidarias, no bastan a hacer de él el primer poeta italiano" ("Gabriel d'Annunzio": 1360-1361).

Aun así, aun no considerándolo el primer poeta italiano, las congruencias entre ellos no faltan: las personalidades literarias de Gabriele D'Annunzio y Amado Nervo se han forjado bajo las mismas luces de la bohemia finisecular y el decadentismo ha alimentado con igual generosidad la pluma de ambos.

Tras estas breves premisas, quizás se perciba de manera menos hostil y escéptica la propuesta de una investigación que tenga el propósito de destacar cierta confraternización de imaginarios en sus narrativas.

El estudio quiere profundizar en el desarrollo literario de la sublimación erótico- estética de la que gozan y padecen las mujeres que infestan los harenes simbólicos de D’Annunzio y Nervo. El foco de atención priorizará el análisis de la representación lírica, en su sentido más amplio, de la Mujer Fatal y de la quimera de la posesión del Ideal, a través de la lectura de dos cuentos fantásticos: El hombre que robó la "Gioconda" de Gabriele D’Annunzio y "La Diablesa” de Amado Nervo.

En ambos cuentos lo fatal reside en el misterio, el erotismo encuentra su razón de ser en el enigma. El anhelo de posesión de la Belleza Absoluta y del Ideal Artístico genera frustración sentimental, espiritual, artística e intelectual en los protagonistas. La posesión erótica de la femineidad idealizada es una quimera. La mujer se convierte en Musa y carnífice.

Pero, veamos, antes de adentrarnos en la lectura de los dos cuentos, cómo, efectivamente, el magma simbólico al que nos estamos refiriendo impregna las páginas de ambos de manera transversal.

Citaré dos párrafos que ejemplifiquen las recurrentes epifanías de la quimera en sendas producciones. Por un lado, hemos un fragmento de El segundo amante de Lucrezia Buti, un texto autobiográfico de autoría dannunziana:

Grande y apasionado evento sigue siendo para mí la hora de la Quimera, cuando justamente mi vida comenzaba a ser mi arte y mi arte comenzaba a ser mi vida. En todos mis sentidos la realidad ya se imprimía con gallardas huellas; pero de todo lo que mi ojo podía ver, de todo lo que mi mano podía tocar, mi espíritu ya extraía símbolos ardientes. ${ }^{2}$ (99)

En cuanto a Amado Nervo, se ha considerado imprescindible mencionar el llamativo final de El Donador de Almas: "Este es el cuento del Donador de Almas que he tenido el placer y la melancolía de contaros. Guardadlo en vuestro corazón, y plegue al cielo que cuando la Quimera llegue hasta vosotros, la acariciéis con humilde espíritu y en alta contemplación, a fin de que no se aleje y hayáis de amarla cuando parta..." (226).

Introduzcamos, ahora, los cuentos.

${ }^{2}$ Traducción propia desde el texto original en italiano: "Grande e appassionato evento rimane per me l'ora della Chimera, quando appunto la mia vita cominciava a essere la mia arte e la mia arte cominciava a essere la mia vita. In tutti i miei sensi la realtà già s’imprimeva con gagliarde impronte; ma da tutto ciò che il mio occhio potea vedere, da tutto ciò che la mia mano potea toccare, il mio spirito già traeva simboli ardenti” (99). 


\section{Gabriele D’annunzio fantástico: El hombre que robó la "Gioconda"}

Empezaré definiendo sumariamente algunas pautas que caracterizan lo fantástico en Gabriele D'Annunzio y me apoyaré, para ello, a un estudio de Lucio D'Arcangelo, quien subraya que la narrativa de tipo fantástico es, en la obra del escritor, "una experiencia episódica"3 (9), aunque significativa.

D'Arcangelo destaca dos etapas en la escritura fantástica de D'Annunzio: una primera, juvenil, en estado embrionario, que se manifiesta en algunos detalles narrativos de los cuentos de Parábolas y novelas y otro, más maduro, que empieza con La Leda sin cisne para desarrollarse plenamente en El hombre que robó la "Gioconda" (9).

Lo sobrenatural en los cuentos dannunzianos está estrictamente vinculado a las leyendas cristianas, al imaginario religioso que impregnaba y sigue impregnando las tierras de Abruzos. Al mismo tiempo, paradójicamente, casi todos sus cuentos siguen dialogando con la herencia del verismo, un verismo mitigado, eso sí, pero vigente.

Sigo citando a D'Arcangelo: "Lo fantástico surge de un efecto sensorial: un apoyarse a los límites de la realidad, donde esta es más dudosa y porosa" (14). El narrador dannunziano juega con la percepción esperpéntica de lo real y, lo hace, también, "manipulando", digamos, los elementos de la naturaleza a su favor y antojo.

El estudioso menciona el ejemplo de "Las crisantemas", de la sección "Fantasías" de Parábolas y novelas. Protagoniza el cuento un poeta moribundo, en una condición de debilidad física y excitabilidad. Su alma es habitada por una "inquietud misteriosa" (14) y la luz del jardín abandonado en el que se halla brilla "extraña y sobrenaturalmente" (15). El poeta, sugestionado, no puede no pensar en Percy Shelley y en aquel verso que pintaba las alumbrantes "miradas de las flores" (15). Esas flores, las crisantemas, recogidas por el poeta en su delirante paseo hacia la muerte, resultan ser flores letales, las mismas flores "extrañas, misteriosas, aristocráticas" que dan título al homónimo cuento de juventud de Amado Nervo (Nervo: 137). Este escribe de la crisantema: "No solo es la predilecta de los grandes; es también la predilecta de los poetas. No es solo la flor heráldica; es también la flor... de los espíritus" (138). El narrador argumenta su afirmación contándonos un "hecho del todo auténtico":

Un poeta francés muy conocido buscaba, como todos los poetas, la realización de un ideal imposible: quería a la mujer única, a la mujer toda belleza, toda ternura y toda amor, y esperándola transcurría su vida, y deseándola corrían los años sin hallarla.

Un día, sin embargo, el destino se compadeció del loco, y este, en una asamblea espírita, vio surgir ante él una mujer de divina hermosura, etérea, diáfana, como hecha de luz de estrella, de perfume y de sueño... Acercóse al poeta, y le dijo:

Yo te amo; [...]; soy tuya -. Y sonriendo dulcemente, ofreció a su amado como prenda de su cariño inmortal... un haz de crisantemas doradas... Momentos después, la sublime aparición se esfumó en la media luz de la estancia [...]. (138)

Sigamos con D'Annunzio. En la Leda sin cisne, el narrador nos hace partícipes de su punto de observación alquímico y esperpéntico: "Nuestra vida es una obra mágica, que rehúye el reflejo de la razón y cuanto más plena más se le aleja, actuada por ocultamiento $\mathrm{y}$, a menudo, contra el orden de las leyes aparentes" (39). ${ }^{4}$ Entre las páginas de este cuento hallamos "La leyenda del hombre sin mirada" que nos conduce directamente hacia El hombre que robó la "Gioconda".

La leyenda, que hace de pórtico al cuento, narra la historia de un hombre que, en la adoración contemplativa del sagrado retrato de una Virgen, debido al intenso fervor de su mirada, le infundió vida al lienzo, quedándose ciego.

Lucio D'Arcangelo considera El hombre que robó la “Gioconda” "el único texto de D'Annunzio consciente y rotundamente fantástico" (9) y afirma que "pocas veces D'Annunzio ha expresado tan claramente la distancia entre arte y mundo real" (20).

Entremos en materia.

Los protagonistas de la historia son el mismísimo Gabriele D'Annunzio y un pintor alquimista. Este último se propone robar el famosísimo cuadro de Leonardo Da Vinci para hacer que la Gioconda cobre vida.

\footnotetext{
${ }^{3}$ Traducción propia desde el original en italiano. De ahora en adelante solo se citará el ensayo en castellano.

${ }^{4}$ Traducción propia del texto original: La Leda senza cigno.
} 
D’Annunzio, quien en la obra se define a sí mismo como "el más noble amante de la Mona Lisa" (35) consigue recibir en su casa a la prodigiosa encarnación. El incrédulo poeta cree poder poseer "el amor de su sueño, el amor de su poesía. El enigma de las líneas sobre las cuales se había afanado su espíritu, [...] transformado en vida respirante, en carne sensible" (51- 52).

Sin embargo, una trágica realidad hiere al poeta: La Gioconda "ya no sonríe, el adorado "gesto leonardesco se descompone" (50) en vida. Desesperado gime: "Te he buscado a ti en todas las amantes. He buscado en todos los labios voluptuosos tu sonrisa. Estás viva y respiras. Estás viva y hablas. ¿O estabas más viva cuando no respirabas? ¿Cuándo no hablabas?”. En balde la implora: “¡Responde! ¡Responde! ¡Déjate conocer! Déjate poseer!" (53). En cuanto el poeta delirante intenta acercarse, su amada ideal desvanece y solo queda la ardiente frustración.

\section{Amado Nervo fantástico: "La Diablesa"}

Aunque quizás sobre la premisa, cedo ante ella: la prosa de Amado Nervo ha sufrido cierta marginación por parte de la crítica, deslumbrada, no sin razones, por su poesía.

Y, aunque, Ana Vigne Pacheco, afirme, con acento algo demasiado confiado que "a Nervo se le ha otorgado el lugar que se merece dentro de la prosa modernista, o sea el de un precursor de la literatura fantástica y el de un ágil y ameno cronista" (13); Francisco González Guerrero y Francisco Tovar Blanco coinciden en constatar que las obras en prosa de Amado Nervo se hallan todavía lamentablemente en un espacio intersticial y lo cuestionan.

Guerrero, en su introducción a las Obras Completas, reivindica el valor literario de dichas obras y precisa que "por su gracia voluble y sugestiva, semejan un eco de su poesía" (29) y, encuentra en la prosa de Nervo: "la del hombre que cautiva a los demás por las virtudes órficas del canto, cualesquiera que sean las formas de su expresión" (9); "que revela a veces al afán de cada día, (y) también suele mostrar el hallazgo precioso, como queda la perla del rocío aprisionada en la urdimbre de la araña" (29); "[...] una diversa manera del divagar poético, un ocasional descanso de la rima y del ajuste de sílabas y acentos" (9).

Tovar Blanco, por su parte, profundiza en los escritos fantásticos, celebrando sus méritos. En su ensayo sobre las "Visiones fantásticas en la prosa literaria de Amado Nervo" analiza "La Diablesa" y El donador de almas. Me centraré en el primer cuento.

De la carta- prólogo, firmada por el mismo escritor quien se suma al mismo juego meta- narrativo de D'Annunzio en El hombre que robó la "Gioconda", se aprende que el motivo de inspiración de "La Diablesa" es una mujer concreta, "una soberbia dama" (129), una cierta Elena. Al destinatario de la carta, don Manuel Larrañaga Portugal, Nervo pregunta si "el boceto es fiel", "si es ella" (129). Lo veremos.

El protagonista es "el joven don Jaime Álvarez de la Rosa, soltero, de treinta y tres años, rico, solo y fuerte. [...] Inteligente, artista, hombre de mundo, jamás se negó un placer, por costoso que fuese" (130). Nada lo diferencia del Andrea Sperelli dannunziano.

Don Jaime, atormentado por el tedio, formula una peculiar invocación, pide al Diablo un deseo: Elena. "Elena es el ideal encarnado; la Venus de Cleomeno con el alma de Ofelia; la Venus mortal, con las ternuras apasionadas de Eros, la coquetería inmensa de Ninón, el valor de Judith, la constancia de Penélope, la voz de las sirenas mitológicas, los refinamientos de Aspasia, [...]" (130), y, extraordinariamente, la consigue. El incrédulo le pregunta: “- ¿Eres mía?”; “- Tuya” (133-134), contesta Elena sin titubear.

El Diablo no pide el alma del afortunado hombre a cambio, pero impone una tremenda condición: "Elena desaparecerá en el mismo momento en que engañe a su amante, porque el modelo ideal no incluye la fidelidad" (Tovar Blanco).

Otra vez la quimera de la posesión frustrada.

Hermosa coincidencia, la que hermana a las dos Elena, ambas infieles: la Diablesa de Don Jaime Álvarez de la Rosa y la evanescente amante del héroe dannunziano, Andrea Sperelli. Cito la página 24 de la novela $E l$ placer:

La certeza de la posesión le rehuía. Elena le parecía una mujer nueva, nunca gozada, nunca abrazada [...]. El enigma casi diré plástico de su belleza era aún más oscuro y atrayente. [...] había 
en sus ojos y en su boca un singular contraste de expresión apasionada, intensa, ambigua, sobrehumana, que solo algún moderno espíritu, impregnado de toda la profunda corrupción del arte, ha sabido infundir en tipos de mujeres como Mona Lisa o Nelly O' Brien. ${ }^{5}$

Y, para concluir, un detalle interesante: en el apellido de Don Jaime se intuye una sugerencia. El placer de Gabriele D'Annunzio junto con el Triunfo de la Muerte y El inocente, a partir de 1895, formaría parte de una trilogía titulada Las novelas de la Rosa. Quizá sea otra simple coincidencia.

\section{Cierre}

Sobre el "círculo de la sensualidad" que rodea a los personajes dannunzianos Gonzalo Zaldumbide aclara algo interesante que también podría involucrar a los protagonistas de las obras recién mencionadas de Nervo. Al hablar de la sublimación erótico- estética de la mujer y de la fatal quimera de su posesión, afirma:

No se trata aquí del puro y simple deseo sensual que halla su satisfacción, por lo menos momentánea, en el orgasmo físico; que se apaga en la saciedad, pero renace luego de sus cenizas y recomienza sus vicisitudes dentro del círculo en donde alternan la tentación y el hastío. Se trata de pasiones exacerbadas, sutilizadas con arte sapiente, con refinamientos de esteta, que encuentran en la posesión de la mujer un placer violento, pero falaz; porque tal posesión deja insatisfechas e irritadas otras necesidades, las cuales no pueden hallar en aquel placer la felicidad ideada y esperada. (81-82)

Tras la lectura de los cuentos analizados en esta sede parece quedar al descubierto esa confraternización de imaginarios vislumbrada inicialmente. Y, por muy reacio que se mostrara Amado Nervo frente a los voluptuosos excesos estéticos del "Vate", resulta innegable constatar que al menos algunos de sus personajes fueron poseídos por el espíritu de Andrea Sperelli.

Aun así, la honestidad intelectual impone destacar una diferencia fundamental. Como subraya Francisco González Guerrero, Nervo "nunca se vanaglorió de un estilo deslumbrador y d'annunziano" porque "el secreto de su estilo se encontraba en el extremo contrario: en el de la sobriedad y la discreta elegancia" (31).

\section{Referencias bibliográficas}

D'Annunzio, Gabriele (1947). Il secondo amante di Lucrezia Buti. Milano: Mondadori.

D'Annunzio, Gabriele (1976). La Leda senza cigno. Cles: Arnoldo Mondadori Editore.

D'Annunzio, Gabriele (2010). Il piacere. Roma: Newton Compton editori.

D'Annunzio, Gabriele (2018). L'uomo che rubò la "Gioconda". Chieti: Marino Solfanelli Editore.

D'Arcangelo, Lucio (1990), "D'Annunzio 'fantastico"”, en D'Annunzio in America Latina. Ciclo di convegni su D'Annunzio e le letterature moderne. Chieti: Marino Solfanelli Editore, pp. 7-20.

Meregalli, Franco (1988), “D'Annunzio nella cultura iberica e iberoamericana", en D'Annunzio a cinquant'anni dalla morte. Atti dell'XI Convegno internazionale di studi dannunziani, vol. II, maggio, pp. 647-657.

Nervo, Amado (1973). Obras completas, Tomo I, Prosas. Ed. Francisco González Guerrero. Madrid: Aguilar.

Tovar Blanco, Francisco (2010), "Visiones fantásticas en la prosa literaria de Amado Nervo". Alicante: Biblioteca Virtual Miguel de Cervantes.

Disponible en: http://www.cervantesvirtual.com/nd/ark:/59851/bmcww801 .

Vigne Pacheco, Ana, "Las minificciones de Amado Nervo".

Disponible en: http://www.amadonervo.net/narrativa/minificciones01.html .

Zaldumbide, Gonzalo (1916). La evolución de Gabriel D'Annunzio. Madrid: Editorial América.

\footnotetext{
${ }^{5}$ Traducción propia; aquí la cita original: "La certezza del possesso gli sfuggiva. Elena gli pareva una donna nuova, non mai goduta, non mai stretta. [...] L'enigma quasi direi plastico della sua bellezza era ancor più oscuro e attirante. [...] aveva negli occhi e nella bocca un singolare contrasto di espression passionata, intensa, ambigua, sopraumana, che solo qualche moderno spirito, impregnato di tutta la profonda corruzione dell'arte, ha saputo infondere in tipi di donna come Monna Lisa o Nelly O'Brien."
} 